\title{
Vascular Augmentation in Renal Transplantation: Supercharging and Turbocharging
}

\author{
Euicheol C. Jeong ${ }^{1}$, Seung Hwan Hwang ${ }^{2}$, Su Rak Eo ${ }^{2}$ \\ ${ }^{1}$ Department of Plastic Surgery, SMG-SNU Boramae Medical Center, Seoul; ${ }^{2}$ Plastic and Reconstructive Surgery, Dongguk University \\ Medical Center, Dongguk University College of Medicine, Ilsan, Korea
}

The most common anatomic variant seen in donor kidneys for renal transplantation is the presence of multiple renal arteries, which can cause an increased risk of complications. Accessory renal arteries should be anastomosed to the proper source arteries to improve renal perfusion via the appropriate vascular reconstruction techniques. In microsurgery, 2 kinds of vascular augmentation methods, known as 'supercharging' and 'turbocharging', have been introduced to ensure vascular perfusion in the transferred flap. Supercharging uses a distant source of the vessels, while turbocharging uses vascular sources within the same flap territory. These technical concepts can also be applied in renal transplantation, and in this report, we describe 2 patients who underwent procedures using supercharging and turbocharging. In one case, the ipsilateral deep inferior epigastric artery was transposed to the accessory renal artery (supercharging), and in the other case, the accessory renal artery was anastomosed to the corresponding main renal artery with a vascular graft (turbocharging). The transplanted kidneys showed good perfusion and proper function. No cases of renal failure, hypertension, rejection, or urologic complications were observed. These microsurgical techniques can be safely utilized for renal transplantation with donor kidneys that have multiple arteries with a lower complication rate and better outcome.

Keywords Reconstructive surgical procedures / Regional blood flow / Tissue and organ harvesting

\author{
Correspondence: Su Rak Eo \\ Department of Plastic and \\ Reconstructive Surgery, Dongguk \\ University Medical Center, Dongguk \\ University College of Medicine, 27 \\ Dongguk-ro, Ilsandong-gu, Goyang \\ 10326, Korea \\ Tel: $+82-31-961-5782$ \\ Fax: +82-31-961-7347 \\ E-mail: sreodoc@gmail.com
}

No potential conflict of interest relevant to this article was reported.

\section{INTRODUCTION}

Vascular augmentation (VA) is a subsidiary procedure for enhancing flap viability in the case of compromised blood perfusion. Various techniques have been developed, such as arterial 'supercharging' and 'turbocharging,' venous superdrainage, delayed procedures, and medical agents for vasodilation $[1,2]$. Supercharging and turbocharging are the most commonly performed microvascular procedures for enhancing arterial inflow at the suspended flap periphery. To obtain additional blood supply, supercharging uses a distinct distant source of vessels, whereas turbocharging uses vascular sources within the same flap territory. They have been successfully applied in various skin flaps [2-8].

VA can also be applied in the transplantation of organs that need additional vascular anastomosis. Although renal transplantation is now a well-established procedure, surgeons sometimes encounter abnormal vascular patterns in the donor kidneys. Accessory renal arteries in the donor kidney are one such problem, with a rather high prevalence that ranges from $2 \%$ to $20 \%$ 
$[9,10]$. The presence of multiple renal arteries in the donor kidney may lead to a longer operative time due to requiring a more complicated dissection, and the ischemic time is also longer because of the requirement for a more complex implantation, with an increased risk of vascular complications [11]. The authors, as plastic surgeons, understand the need for VA techniques, such as supercharging and turbocharging, for improving the viability of free tissue transfer. In this report, we present the application of a similar concept in renal transplantation when multiple renal arteries are present in the kidney, introducing cases and reviewing the previous literature.

\section{CASES}

Two patients with abnormal renal arteries were referred to the Department of Plastic Surgery for microsurgical anastomosis at Dongguk University Hospital between July 2013 and August 2014. Accessory renal arteries were incidentally found by the general surgery team during the renal transplantation procedure. One patient had an accessory renal artery in the posterior upper pole, and the other had one in the lower pole.

We applied 2 microsurgical techniques separately for VA of the renal arteries: turbocharging and supercharging using the deep inferior epigastric artery (DIEA) (Fig. 1).

(1) Supercharging: a procedure was designed to result in the usual pair of the accessory renal artery and vein, supplied from another artery and vein. The proximal end of the ipsilateral DIEA was ligated and transferred to perform an end-to-end anastomosis to the accessory lower polar renal artery.

(2) Turbocharging: surgical anastomosis was performed between a main renal artery and an accessory renal artery. End-toside anastomosis of the arterial graft from the DIEA to the main renal artery and end-to-end anastomosis to the accessory renal artery were performed under a microscope.

Accessory renal arteries were separately noted in the operating field in the posterior upper (case 1) and lower (case 2) portions of the transplanted kidney. Arterial anastomosis was performed under microscopic magnification with the conventional technique, using 10-0 Ethilon sutures.

\section{Case 1}

A 43-year-old man was referred to our hospital because of endstage renal disease (ESRD) due to immunoglobulin A nephropathy. His right kidney was transplanted from a living donor. In the donor kidney, the main renal artery was $5 \mathrm{~mm}$ in diameter and the accessory renal artery was $2 \mathrm{~mm}$ in diameter in the posterior upper pole. Microsurgical anastomosis of the main renal artery was performed by the general surgical team. However, the posterior upper pole remained purple due to poor blood supply. We harvested the ipsilateral DIEA for supercharging the transplanted kidney. The DIEA was transected at the branching point from the external iliac artery and transferred for an additional microsurgical anastomosis to the posterior upper segment of the accessory renal artery by end-to-end anastomosis. As soon as the additional anastomosis was carried out, the pole recovered its color (Fig. 2).

\section{Fig. 1. Supercharging and turbocharging techniques of vascular augmentation}

(A) Supercharging technique: the proximal end of the ipsilateral DIEA was used for end-to-end anastomosis to the small lower polar renal artery. (B) Turbocharging technique: an end-to-side anastomosis of the accessory renal artery to the main renal artery was performed with an ipsilateral DIEA graft. RA, renal artery; RV, renal vein; U, ureter; aRA, accessory renal artery; DIEA, deep inferior epigastric artery.
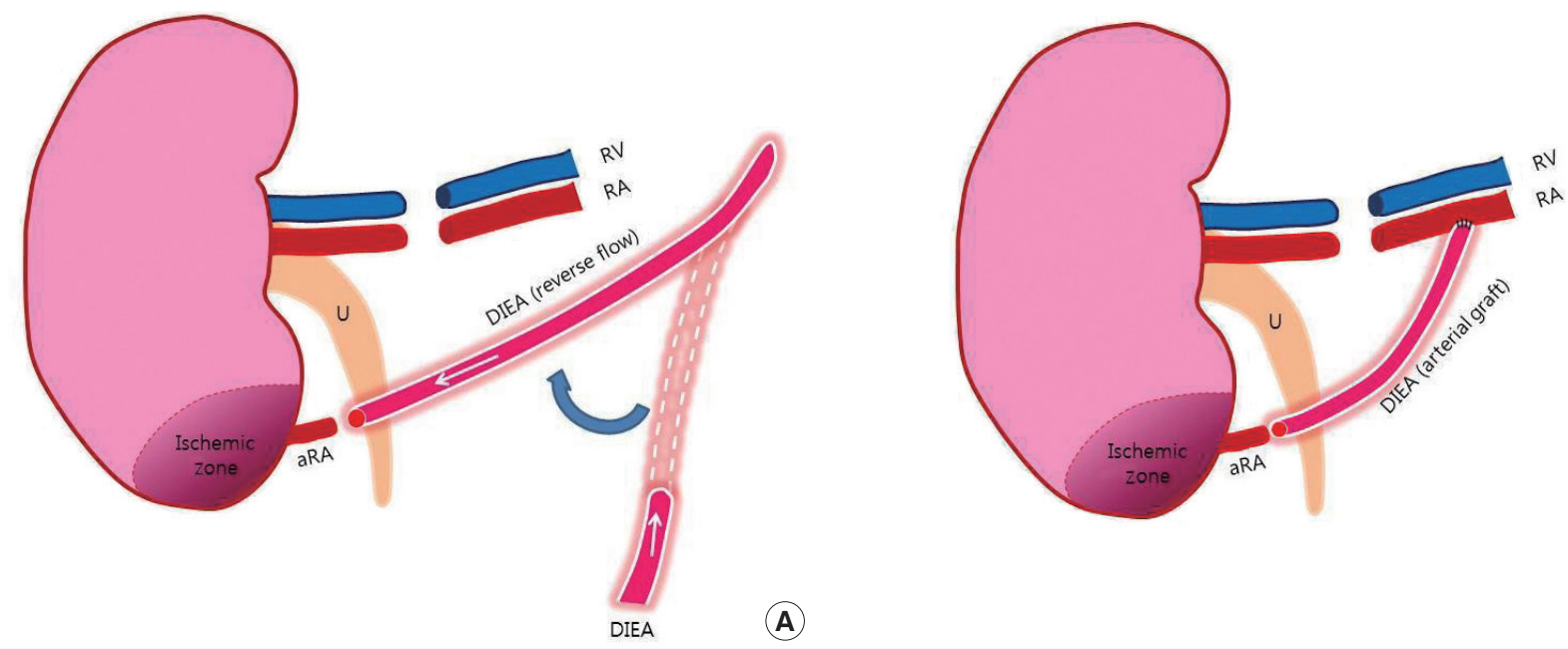


\section{Fig. 2. Case 1, supercharging technique}

(A) Isolation of the deep inferior epigastric artery. (B) Supercharging arterial augmentation by an anastomosis between the deep inferior epigastric artery and the accessory renal artery of the donor kidney. DIEA, deep inferior epigastric artery; Rt., right.
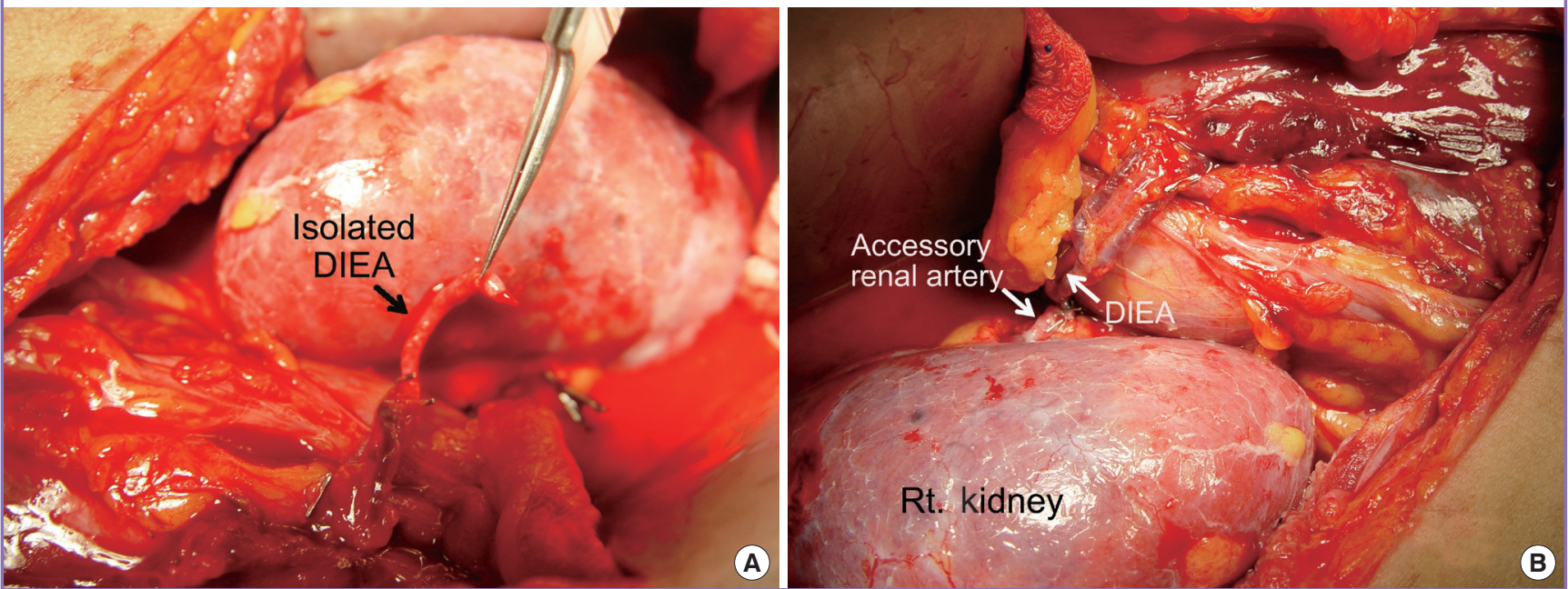

\section{Fig. 3. Case 2, turbocharging technique}

(A) Deep inferior epigastric artery graft. (B) Turbocharging arterial augmentation with an ipsilateral deep inferior epigastric artery graft. The accessory artery was found in the operative field and vascular augmentation was performed by anastomosis with a deep inferior epigastric artery graft. DIEA, deep inferior epigastric artery; Rt., right.

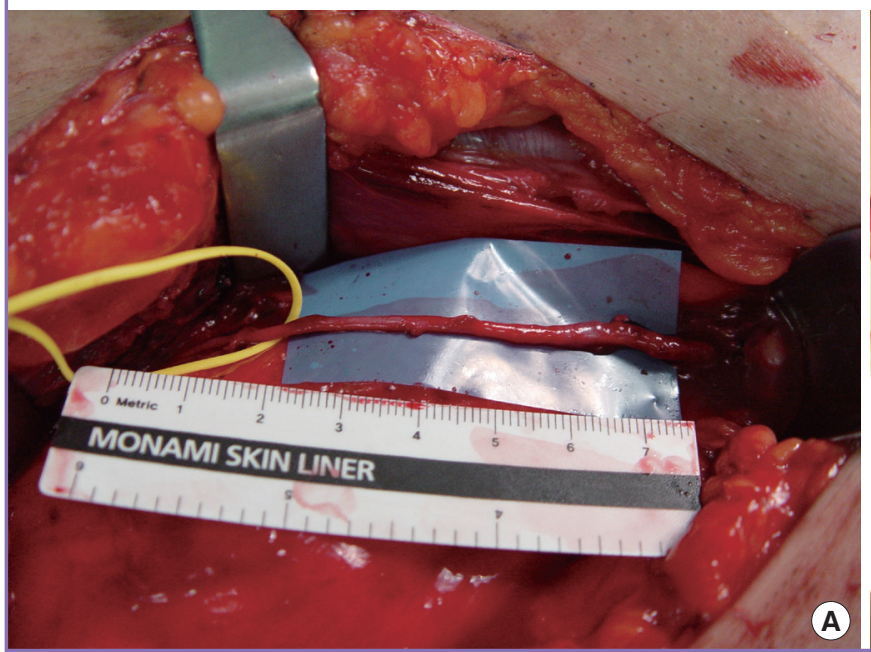

\section{Case 2}

A 54-year-old man who suffered from ESRD caused by membranous glomerulonephritis was referred to our hospital. His right kidney was transplanted from a donor who experienced brain death. The main renal artery from the donor was $8 \mathrm{~mm}$ in diameter; the accessory renal artery was $1 \mathrm{~mm}$ in diameter, and was found in the lower pole. Microsurgical anastomosis of the main renal artery was performed for the arterial graft by the general surgery team, but the color of the lower pole remained purple, in contrast to most past procedures. We harvested the DIEA (10 cm in length) on the ipsilateral side. The DIEA was interposed between the accessory renal artery in an end-to-end pat-

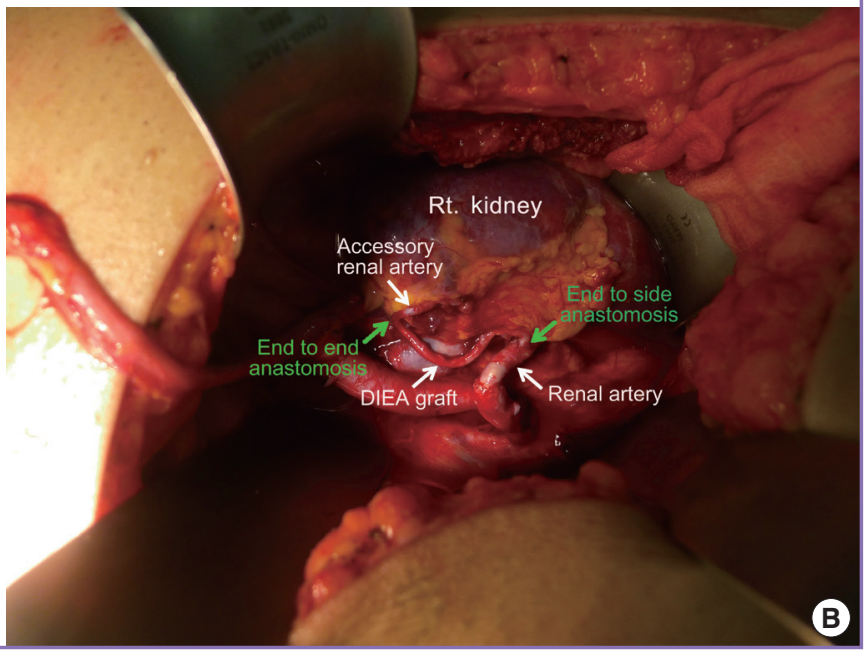

tern, and the main renal artery in an end-to-side pattern (Fig. 3). In both cases, the operation was carried out successfully. Good perfusion of the transplanted kidney was confirmed by Doppler ultrasonography and by the change in color immediately after the vascular anastomosis. Graft survival was excellent, without any rejections or complications (major or minor), and the patients were discharged uneventfully. Follow-up outpatient laboratory tests were conducted.

\section{DISCUSSION}

The first kidney transplantation was performed in 1954 [12], 
and it is now one of the most commonly performed transplant operations. Although postoperative complications, such as vascular thrombosis, stenosis, pseudoaneurysm, and arteriovenous fistula formation in the surgical field, can threaten the transplanted kidney [13], the procedure still shows a satisfactory success rate with advances in immunology. Additionally, technical issues have been overcome, because the renal artery and vein are sufficiently large in diameter for vascular surgeons to anastomose. However, the possibility of encountering anatomically unexpected renal vasculature must be considered preoperatively by vascular surgeons. Abnormalities in the renal vasculature has been commonly reported in the literature $[9,10]$.

Encountering renal vascular anatomical variants is not uncommon in renal transplantation. Hypoplasia or atresia of the iliac arterial system, supernumerary and early branching arteries and veins, superior polar renal arteries, and circumaortic or retroaortic renal veins have been reported $[9,10,13,14]$. The accessory renal artery has its own vascular territory, although it may only encompass a small portion of the kidney, and its needs supplementary blood flow to prevent necrosis. Failing to observe the incidental operative presence of such a structure could cause the loss of renal function due to the highly vascular nature of the tissue. Therefore, accessory vascular pedicles must be obtained to guarantee viability in cases such as ours [11]. With the development of imaging techniques such as computed tomographic (CT) angiography, multidetector CT, and multislice spiral CT angiography, incidental operative abnormalities of the renal arteries are no longer perceived to be harmful enough to prevent kidney donation [15].

The presence of multiple renal arteries may result in a longer operative time because a more complicated dissection is required. The requirements of bench vascular reconstruction and more complex implantation lead to additional ischemic injuries and subsequent reperfusion injuries [11].

The development of new microsurgical techniques has led to the emergence of new options, such as the supercharged and turbocharged flap techniques [2]. These are 2 kinds of VA for improving the vascular circulation in the transferred flap. Supercharging is the augmentation of the vascularity of a flap by anastomosing distant vessels to the flap, whereas turbocharging is the anastomosis of the vascular source of the flap to the flap itself.

Several techniques for bench or in situ reconstruction of multiple renal arteries to reduce the incidence of complications have been described, including end-to-side anastomosis of the smaller artery to the main artery; side-to-side anastomosis of similarsized arteries; and anastomosis to the recipient internal, external, or common iliac and hypogastric arteries. The proximal and distal ends of the inferior epigastric artery have also been used successfully for end-to-end anastomosis to small polar vessels with arterial inflow inferiorly from the femoral artery and superiorly from the internal mammary artery. These technical developments have expanded the pool of cadaver, living-related, and living-unrelated donors [11].

This array of possible methods often causes surgeons confusion. The complicated nature of vascular reconstruction can cause the prolongation of ischemic time in the donor kidney, so sizeable vessels have been selectively used in vascular reconstruction, such as the saphenous veins or the branches of iliac vessels, to the extent that it is possible without a microscope [11]. Innovative vascular reconstruction methods for improving the vascular circulation of skin flaps have been introduced in the clinical field of plastic and reconstructive surgery. These techniques have mostly been undertaken in small vessels, with a surgical microscope in situ, do not take up a significant amount of time, and their variability has led to the concepts of supercharging and turbocharging. Since these techniques reflect conceptual breakthroughs, they may help lead to the development of novel innovative methods, in addition to promoting skillful microsurgical techniques. Supercharging is when the kidney is supplied by anastomosing another vessel, such as the DIEA, and turbocharging is the anastomosing of the renal artery of the kidney to itself using avascular graft. Although the results of this study cannot show which procedure has better outcomes, conducting additional studies to qualify and quantify these 2 VA techniques could help in choosing between them in the surgical field.

Microsurgical VA enhances reliability and ensures a more predictable outcome. This technique permits sufficient blood supply to the recipient flap or organ, which eventually prevents tissue necrosis. We suggest VA in suitable cases because it does not require sophisticated surgical techniques or a prolonged operative time. We suggest that this concept can be widely applied in the field of surgery, including renal transplantation.

\section{REFERENCES}

1. Fukushima J, Inoue Y, Kiyokawa K, et al. Effects of shortterm venous augmentation on the improvement of flap survival: an experimental study in rats. J Plast Reconstr Aesthet Surg 2012;65:650-6.

2. Civelek B, Kargi E, Akoz T, et al. Turbocharge or supercharge? Plast Reconstr Surg 1998;102:1303.

3. Hashimoto I, Abe Y, Morimoto A, et al. Limb salvage and vascular augmentation by microsurgical free flap transfer for treatment of neuropathic diabetic foot ulcers. J Med Invest 
2014;61:325-32.

4. Numajiri T, Sowa Y, Nishino K, et al. Does a vascular supercharge improve the clinical outcome for free jejunal transfer? Microsurgery 2013;33:169-72.

5. El-Mrakby HH, Milner RH, McLean NR. Supercharged pedicled TRAM flap in breast reconstruction: is it a worthwhile procedure. Ann Plast Surg 2002;49:252-7.

6. Takagi S, Oyama T, Yamazumi K, et al. Vascular augmentation of an extended latissimus dorsi myocutaneous flap through an intercostal vessel: a preliminary report. J Plast Surg Hand Surg 2013;47:123-5.

7. Komorowska-Timek E, Gurtner G, Lee GK. Supercharged reverse pedicle anterolateral thigh flap in reconstruction of a massive defect: a case report. Microsurgery 2010;30:397400.

8. Sbalchiero JC, de Albuquerque Leal PR, dos Santos CC. Breast reconstruction with a turbocharged transverse rectus abdominis myocutaneous flap on the contralateral perforator. Ann Plast Surg 2014;73:503-8.

9. Daescu E, Zahoi DE, Motoc A, et al. Morphological variability of the renal artery branching pattern: a brief review and an anatomical study. Rom J Morphol Embryol 2012;53: 287-91.

10. Dhar P, Lal K. Main and accessory renal arteries: a morphological study. Ital J Anat Embryol 2005;110:101-10.

11. Kadotani Y, Okamoto M, Akioka K, et al. Management and outcome of living kidney grafts with multiple arteries. Surg Today 2005;35:459-66.

12. Leeson S, Desai SP. Medical and ethical challenges during the first successful human kidney transplantation in 1954 at Peter Bent Brigham Hospital, Boston. Anesth Analg 2015; 120:239-45.

13. Glebova NO, Brooke BS, Desai NM, et al. Endovascular interventions for managing vascular complication of renal transplantation. Semin Vasc Surg 2013;26:205-12.

14. Tamisier D, Melki JP, Cormier JM. Congenital anomalies of the external iliac artery: case report and review of the literature. Ann Vasc Surg 1990;4:510-4.

15. Pozniak MA, Lee FT Jr. Computed tomographic angiography in the preoperative evaluation of potential renal transplant donors. Curr Opin Urol 1999;9:165-70. 